\title{
IUPAC Periodic Table Challenge
}

\section{by Juris Meija, Javier Garcia-Martinez, and Jan Apotheker}

n 2019, the world celebrated the International Year of the Periodic Table of Chemical Elements (IYPT2019) and the IUPAC centenary. This happy coincidence offered a unique opportunity to reflect on the value and work that is carried out by IUPAC in a range of activities, including chemistry awareness, appreciation, and education. Although IUPAC curates the Periodic Table and oversees regular additions and changes, this icon of science belongs to the world. With this in mind, we wanted to create an opportunity for students and the general public to participate in this global celebration. The objective was to create an online global competition centered on the Periodic Table and IUPAC to raise awareness of the importance of chemistry in our daily lives, the richness of the chemical elements, and the key role of IUPAC in promoting chemistry worldwide. The Periodic Table Challenge was the result of this effort.

\section{Periodic Table Challenge}

The Periodic Table Challenge was designed as an online quiz about all 118 chemical elements in order to offer students an opportunity to participate in IYPT2019 without requiring large amounts of effort. For those who wanted to delve a bit deeper, an opportunity to showcase their passion about the Periodic Table was provided in a form of the Nobelium Contest, the second round of the Challenge.

\section{Round 1: The Quiz}

In the first round of the challenge, participants were confronted with 15 multiple choice questions about the chemical elements. These questions were selected by the Task Group members. A query to all IUPAC Divisions and Standing Committees was sent out to

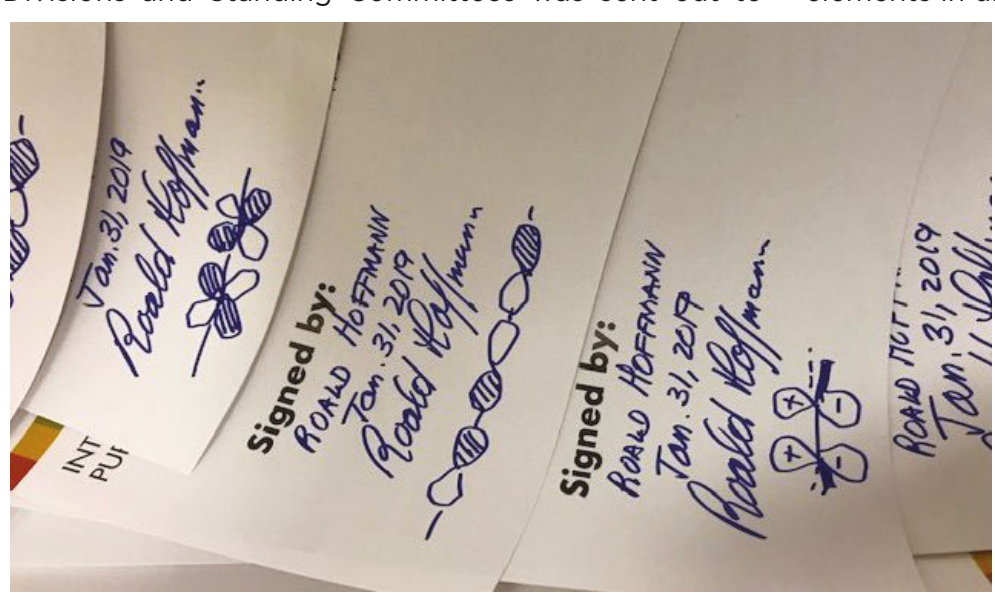

propose questions, and general public was also invited to submit their questions. Overall, questions were received from 20 countries and the Task Group selected the most suitable, edited them, and created new entries until a pool of about 150 questions was achieved involving all chemical elements. Formulating questions about the superheavy elements was a challenge.

The Periodic Table Challenges was conceived and design to highlight the role of IUPAC in supporting, promoting and creating consensus on everything chemistry, most notably in naming and assigning a symbol to chemical elements. This was possible in a number of cases, like for example in the following question:

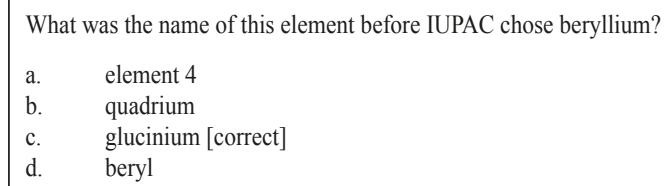

In other cases, elements related to popular culture:

BROMINE What famous TV show features the chemical symbol of bromine?

$$
\begin{aligned}
& \text { a. Breaking Bad [correct] } \\
& \text { b. Band of Brothers } \\
& \text { c. The Big Bang Theory } \\
& \text { d. Prison Break }
\end{aligned}
$$

The questions about chemical elements had a varied level of difficulty. The least-difficult question was about silver (what country is named after this element) whereas a more difficult questions was about carbon (how many moles are there in $12 \mathrm{~g}$ of diamond made of pure carbon-12). Indeed, the timing was perfect for the latter question to be at the center of attention given that 2019 witnessed a new definition of the mole. A majority of entries were about the use of chemical elements in unusual ways to provide a memorable fact

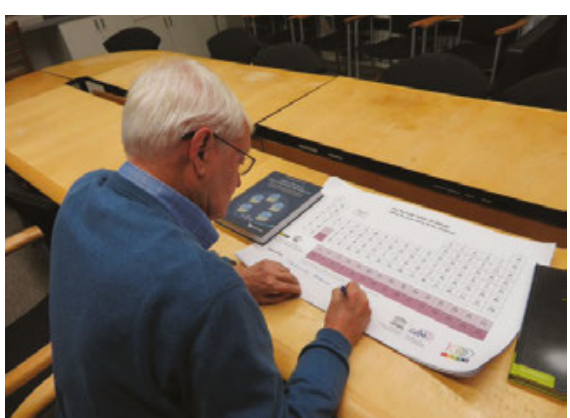

(left) Periodic Tables beautifully signed by Roald Hoffmann (Nobel laureate, 1981) and (above) Joachim Frank (Nobel laureate, 2017) signing them. 


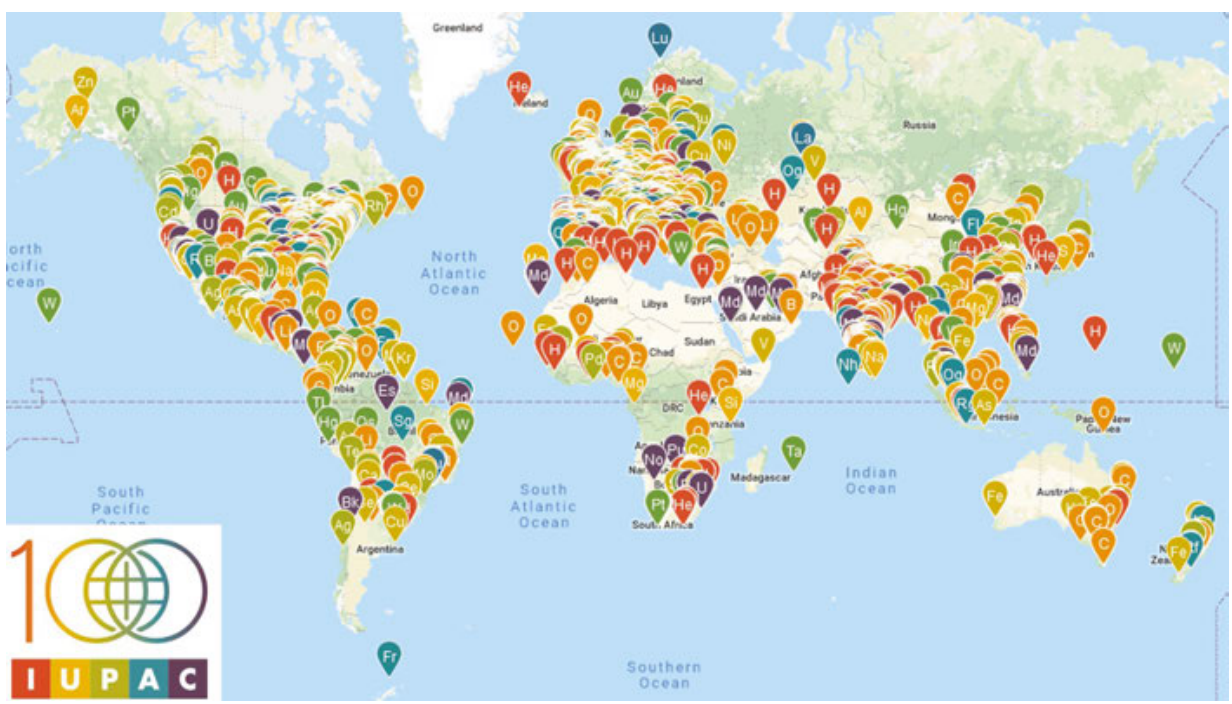

The IUPAC Periodic Table Challenge with element pins representing players from 136 countries/ territories.

for many elements. Thus, thallium was linked to Agatha Christie's The Pale Horse, vanadium was linked to Henry Ford ("but for vanadium there would be no automobiles"), and scandium to tennis rackets.

Upon visiting the welcome page, iupac.org/100/ pt-challenge/, visitors were asked to take the challenge. People were then asked to pick an element as avatar, before taking the 15-question test about the chemical elements. These questions were picked at random from the pool of about 150.

This was done so people could play multiple times and improve their score while learning new things about chemistry and the elements. After answering the questions, each time picking one of the four multiple-choice alternatives, the participant received immediate feedback with explanation of the correct answer. Meanwhile, a tally of the correctly answered questions was also shown during the quiz. After conclusion of the test, participants saw an explanation about the answers to the questions.

If more than $60 \%$ of these questions were answered correctly, participants received a diploma as a PDF file with their name and city. The correct answers to all questions could be found on the internet which further stimulated participants to spend some time online and search for facts about elements in the Periodic Table. We were proud that many school teachers chose to use the PT challenge in their classrooms.

\section{Round 2: The Art Competition}

After correctly answering $60 \%$ of the questions, participants qualified to take part in the second round of the PT Challenge which was dubbed the "Nobelium Contest." The name derives from the fact that we asked chemistry Nobel laureates to sign Periodic Table posters to be given out as prizes. Thirteen chemistry Nobel Laureates joined the Nobelium Contest! We would like to use this opportunity to heartily thank Roald Hoffmann (Nobel Prize 1981), Jean-Marie Lehn (1987), Barry Sharpless (2001), Kurt Wüthrich (2002), Peter Agre (2003), Robert H. Grubbs (2005), Martin Chalfie (2008), Ada Yonath (2009), Robert J. Lefkowitz (2012), Ben Feringa (2016), Sir Fraser Stoddart (2016), Joachim Frank (2017), and Frances Arnold (2018) for their support! They made the Nobelium Contest possible.

After a first round based on the knowledge of the participant of the chemical elements, in this new phase of the Challenge, participants were invited to share their passion and creativity about chemistry. The entries were supposed to highlight the role of the Periodic Table in a creative manner. We received a variety of submissions including videos, poems, songs, and paintings. Entries were categorized into science, art, and outreach or community activities related to the science education and the Periodic Table. We were genuinely surprised and inspired by the dozens of pieces of art that we were receiving from all around the world. A jury as well as popular votes casted for each entry decided who were to receive the special limited edition IUPAC Periodic Tables signed by the Nobel laureates. That was one of the most difficult tasks of this activity, as the quality, originality, and diversity of the entries were truly amazing.

\section{Number of participants in the first round}

One of the most attractive and engaging features in the website of the Periodic Table Challenge, was the world map showing the location of all participants around the world. Pins were emerging from all around the world: from remote islands in Oceania, 
to high-mountains of Nepal and Bhutan, and to many metropolises. Students from every corner of the planet joined and proudly placed their school on the map. We reached 100 countries in the first month of the Challenge. In July, shortly before the IUPAC general Assembly in Paris, Trinidad and Tobago became the 118th country to join the activity thus providing us a symbolic milestone of one country per element. By the end of the year, we reached 136 countries closing with Angkor, Cambodia.

Over the course of the year, more than 8000 players joined in to celebrate the IYPT2019 from every continent and 136 countries/territories. Over 60000 tests were taken and 8000 certificates earned. In some cases whole school classes took part in the challenge. These figures send a clear message that online activities such as this, can make a global impact to promote science education worldwide.

By the end of 2019, India led the country rankings (most correct answers) followed by Canada and USA. We also managed to get participants playing from Antarctica and the Vatican City. However, we were most proud of the geographical diversity we achieved, which included more than 20 countries in Africa and every single country in South America.

A Chinese translation of the PT Challenge was created by the China Digital Science and Technology Museum and the Chinese Chemical Society (chemicaldati. dg.cdstm.cn/admin/api/) in June 2019, and the Chinese version was played over 30000 times over the period of five months.

One of the features of the PT Challenge was that players had to choose an element as their avatar. Throughout the year, a leaderboard indicated the element ranking which participants used as avatar. It is not

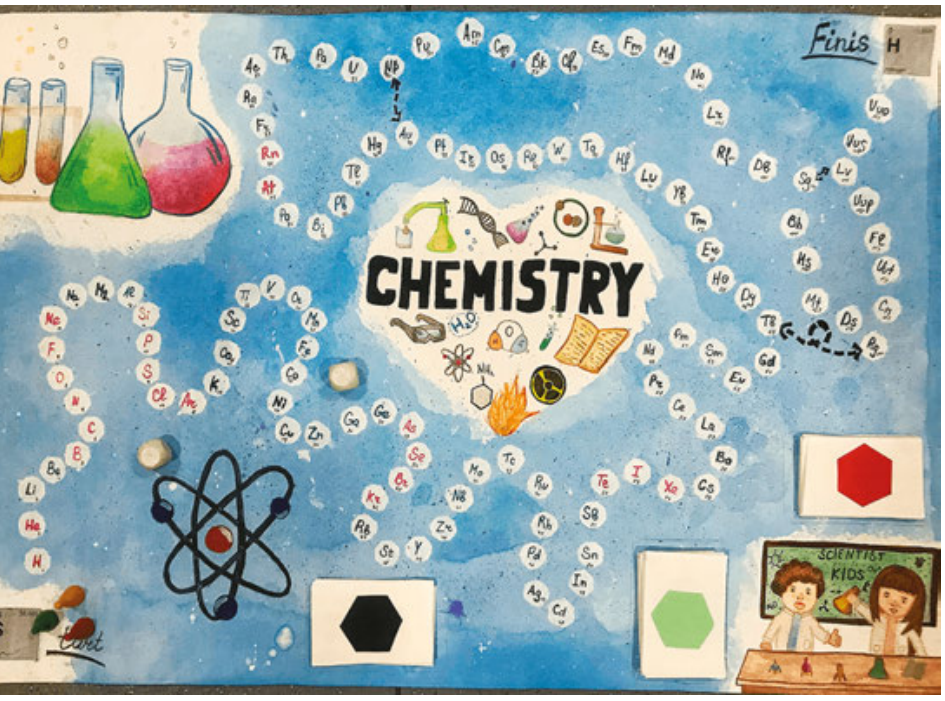

surprising that among the four most popular elements chosen as avatars, you can find elements most closely related to life-hydrogen, carbon, and oxygen. But the hands-down winner, the most popular element of them all, has been mendelevium; a very appropriate recognition of the contribution of this great Russian chemist to the development of the Periodic Table.

Much has been talked about favorite chemical elements and our data shed some light on this matter. For example, recent Nature Chemistry survey of unpopular elements shortlisted protactinium and indeed this element was the least chosen in our Challenge. More interesting, our data revealed some interesting cultural aspects of the Periodic Table: most people who chose to play as francium were from France, most people who chose to play as dubnium were from Russia, most people from Russia played as ruthenium (element named after Russia), most people from New Zealand played as rutherfordium (Rutherford was born there), and most people from Latvia played as livermorium (its symbol is the country code). These observations indicate that players did not see elements as black boxes in the Periodic Table; on the contrary, many had some emotional bond to the elements.

\section{Nobelium contest}

One of the reasons to celebrate the Periodic Table was to provide a platform to show the enthusiasm and pride we all have about the Periodic Table and use this excitement as an opportunity to promote creativity in science. We received all kinds of submissions which varied from educational videos about an element, to

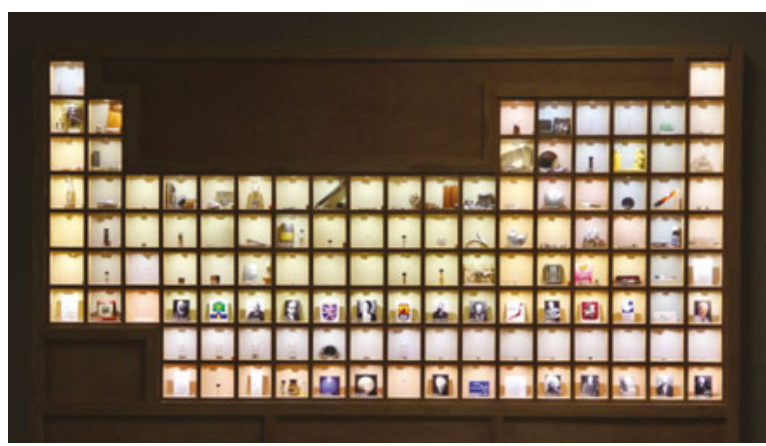


poems, songs, and paintings. Some of the most inspiring entries highlighted the outreach and community service in chemistry and chemistry education.

Several entries were music videos created to highlight the periodic table. Sometimes they were related to festivities, like Christmas. In other cases, very young students were reciting Tim Lehrer's famous element song. Other entries linked the elements to musical notes, making it possible to play the periodic table as a musical instrument. In another case a special periodic table in Morse code was made. We received several stories from Italy some of which were most appropriately inspired from Primo Levi's book The Periodic Table. In other cases, sport analogies were made by linking the Periodic Table with basketball or soccer. Of course the Table was also baked in one way or another.

Over the course of the year, we received over 160 submissions from 30 countries, all of which were hosted online at iupac.org/100/pt-challenge. 30 submissions were selected as Nobelium Contest Winners and further 9 were selected for People's Choice award based on their popularity. The Nobelium Contest entries were viewed over 80000 times throughout the year

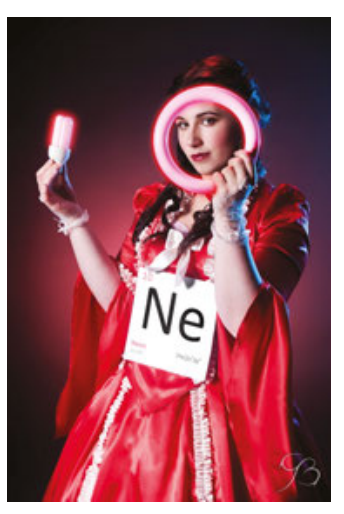
and these winners were all sent the Nobel-autographed IUPAC Periodic Table.

\section{Outlook}

The Periodic Table Challenge has indeed energized the global outreach of IUPAC well beyond its National Adhering Organizations. It succeeded in reaching out to all continents, including Antarctica, and it can lay a claim of

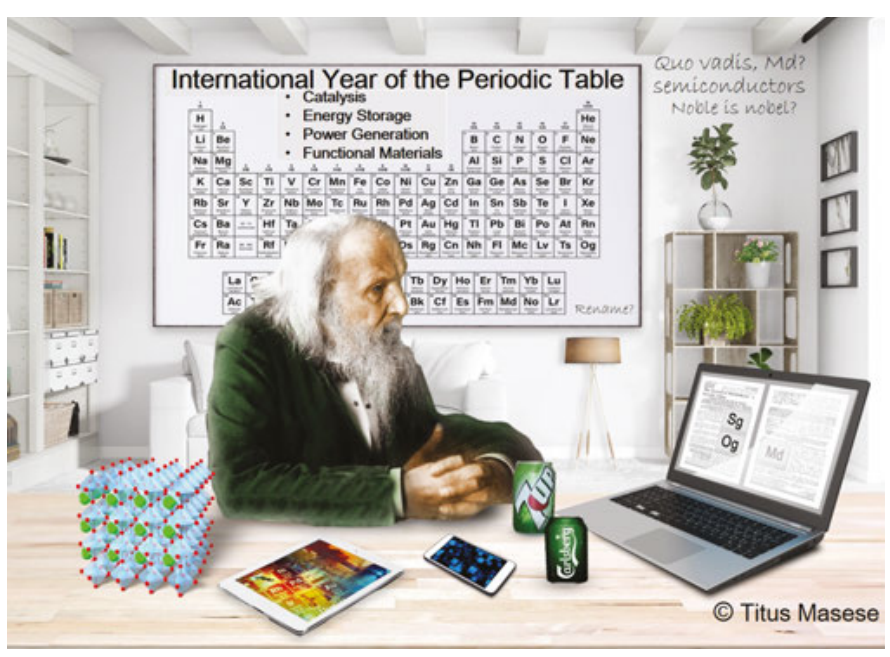

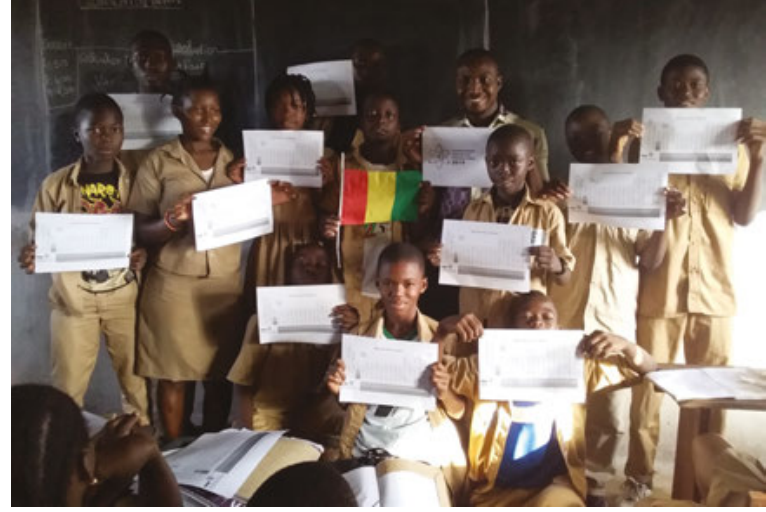

being a truly global contest. Thousands of people from 136 countries had participated thus making the Periodic Table Challenge one of the most popular activities of both the International Year of the Periodic Table and the centenary of IUPAC.

We were impressed by the fact that people took the test on average eight times. It indicates that participants wanted to learn more about other elements and wanted to seize the opportunity to get one of the official IUPAC certificates. Another positive aspect of the PT Challenge was that teachers used the challenge in their classroom, as evidenced by the Nobelium Contest entries.

The Periodic Table Challenge met our expectations and constituted a wonderful way to celebrate the 150th anniversary of the Periodic Table as well as the centenary of IUPAC. This success motivated us to keep the Periodic Table Challenge beyond 2019 as a permanent feature on the IUPAC website. This would involve a regular update of the pool of questions we created in 2018. The Challenge will become one of the outreach activities of IUPAC and a wonderful legacy of 2019. The authors would like to end this brief summary about the Periodic Table Challenge by thanking everyone who made it possible, including IUPAC staff and volunteers, and all the participant who, with their unwavering enthusiasm, have contributed to celebrate the Periodic Table in every corner of the world.

Juris Meija is Research Officer at National Research Council of Canada. He is Titular Member of the IUPAC Inorganic Chemistry Division, Chair of the Commission on Isotopic Abundances and Atomic Weights, and associate member of Interdivisional Committee on Terminology, Nomenclature and Symbols. Javier García Martínez is professor of inorganic chemistry at the university of Alicante in Spain and IUPAC vice-president. Jan Apotheker is professor at University of Groningen, Groningen, Netherlands and chair of the IUPAC Committee on Chemistry Education.

This IUPAC100 activity was developed as project https://iupac.org/project/2017-031-1-050 